\title{
On the structural relations of malachite. II. The brochantite MDO polytypes
}

Frank Girgsdies and Malte Behrens

\section{Supplementary material}

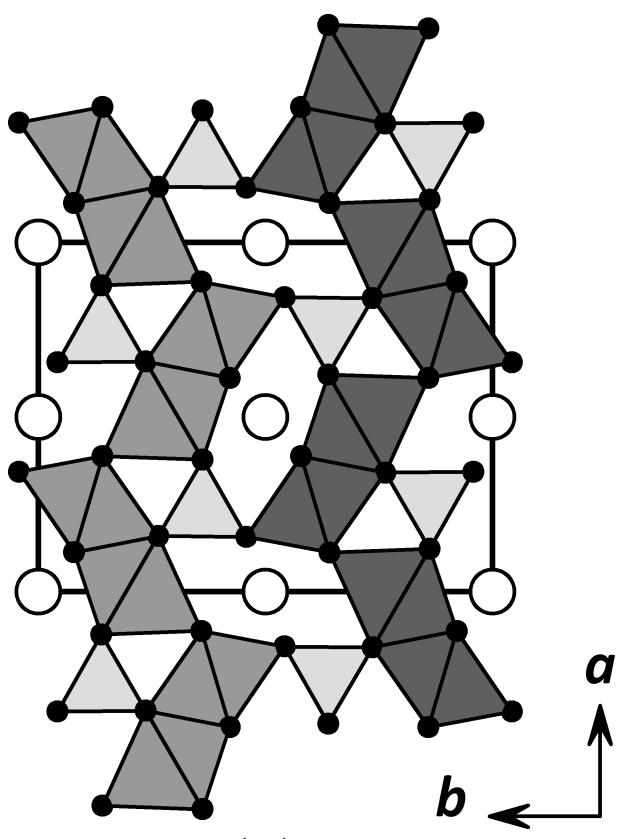

(a)

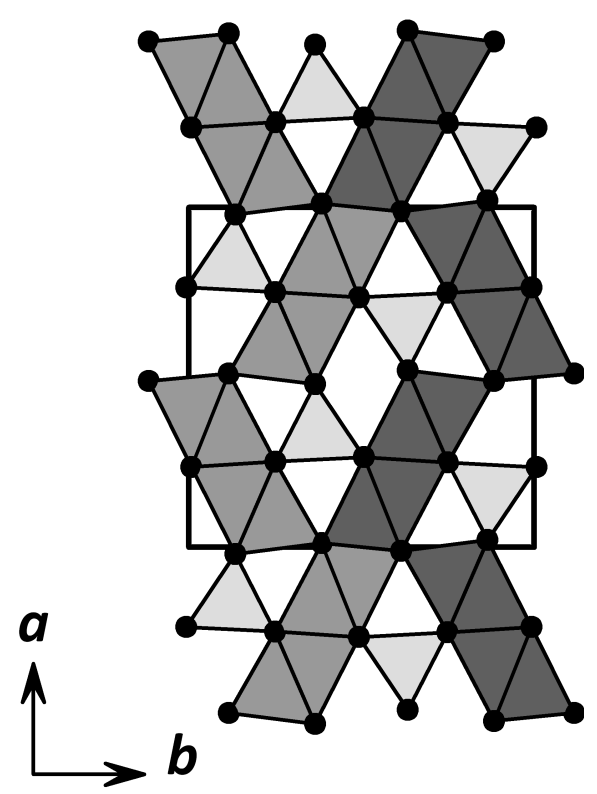

(b)

Figure S1 Comparison of $(a)$ the ludwigite structure $M_{3} \mathrm{O}_{2} \mathrm{BO}_{3}=M_{2}[M]^{\text {channel }} \mathrm{O}_{2} \mathrm{BO}_{3}$ with $(b)$ the warwickite structure $M_{2} \mathrm{OBO}_{3}$. The warwickite structure can be envisioned as being derived from the ludwigite structure by "condensation" of the malachite-like building blocks (medium/dark gray) via shared oxygen atoms (black spheres). The collapse of the "interlayer" space is accompanied by a loss of the $[M]^{\text {channel }}$ sites (white spheres), while the number of linker sites (light gray triangles) remains constant. 